\title{
Obesity Management in Adolescents: Comparison of a Low-Intensity Face-to-Face Therapy Provided by a Trained Paediatrician with an Intensive Multidisciplinary Group Therapy
}

\author{
Catherine Chamay-Weber Nathalie J. Farpour-Lambert \\ Catherine Saunders Gasser Xavier E. Martin Claudine Gal Albane B. Maggio
}

Children's Hospital, University Hospitals of Geneva, Geneva, Switzerland

\author{
Key Words \\ Obesity · Body mass index · Adolescents · Management · Group therapy · Face-to-face \\ therapy $\cdot$ Intensity $\cdot$ Multidisciplinary
}

\begin{abstract}
Objective: This study aimed to compare the effects of a low-intensity face-to-face therapy provided by a trained paediatrician to an intensive group therapy provided by a multidisciplinary team on the BMI of adolescents with obesity. Methods: This longitudinal cohort study included 233 adolescents aged $11-18$ years (mean $13.1 \pm 1.7$ years). Patients and their parents choose either a low-intensity face-to-face therapy or an intensive group therapy (total $88 \mathrm{~h}$ ). Results: At baseline, the mean BMI was $29.4 \pm 4.9 \mathrm{~kg} / \mathrm{m}^{2}$. Within groups changes of BMI zscores were significant at the end of follow-up both in the face-to-face $(-0.2 \pm 0.5)$ and the group therapy $(-0.24 \pm 0.5)$. There was no difference among groups. Younger age (12-14 years), gender, follow-up duration as well as BMI z-score at inclusion were significantly related to BMI z-score changes, independently of the type of intervention. As expected, the face-to-face therapy was far less expensive than the group therapy (USD 1,473.00 \pm 816.00 vs. USD 6,473.00 \pm 780.00 ). Conclusion: A low-intensity face-to-face therapy resulted in similar changes of the BMI z-score of adolescents than an intensive multidisciplinary group therapy. This approach could be easily disseminated in primary care settings with a specific training in obesity care.

(C) 2016 The Author(s)

Published by S. Karger GmbH, Freiburg
\end{abstract}

Catherine Chamay-Weber and Nathalie Farpour-Lambert contributed equally to this article. 


\section{Introduction}

Childhood obesity is one of the most serious public health challenges of the 21st century, and its prevalence is increasing worldwide $[1,2]$. It has a significant impact on both physical and psychosocial health, and put this population at risk of poor health and disabilities in adulthood [3-6]. The treatment of child and adolescents obesity has been the subject of many studies. Multidisciplinary approaches, including nutrition, physical activity and behaviour therapy, are currently recommended [7-9] Family-based cognitive behavioural therapies are also emphasised, being more effective than interventions focused on the child only [9-14]. Stepped-care approaches, which mean increasing the intensity of the treatment with children who do not lose weight, are also recommended. Studies have shown that the more intensive the treatment, the more effective was the intervention, with medium (26-75 h contact time) to high-intensity ( $>75 \mathrm{~h}$ of contact time) therapies being more effective than lower-intensity ones $(<25$ h of contact time) $[9,15,16]$.

Most of these interventions were done in hospital-based units such as paediatric obesity clinics and required multiple health care professionals, which are often beyond the capacity of many clinical settings. Some of them were conducted in a primary care setting and have shown mixed results, with uncertain effect on excessive weight gain $[9,15-20]$. Most of these studies were performed in children under 12 years of age, and there is currently a research gap on feasible and effective obesity management strategies to treat adolescents. They may require special developmental considerations and therapeutic educational approaches, and it is still unclear if therapeutic programmes developed for children are also effective for adolescents $[21,22]$. Their availability to attend intensive programmes may indeed be an important limitation for their participation.

The main purpose of this study was to compare the effects of two different types of interventions on the BMI of obese adolescents: a low-intensity face-to-face therapy provided by a trained paediatrician and an intensive group therapy provided by a multidisciplinary team.

\section{Material and Methods}

\section{Study Design and Subjects}

The present study was designed as a longitudinal cohort study including 233 adolescents with obesity (BMI > 97 percentile, WHO references) aged 11-18 years (mean \pm standard deviation (SD) $13.1 \pm 1.7$ years) who visited the Paediatric Obesity Clinic of the University Hospitals of Geneva (HUG) between January 2008 and June 2012. Adolescents with obesity were referred by paediatricians, general practitioners, school nurses, families or by the staff of the Department of Child and Adolescent of the HUG.

After an initial assessment by the paediatrician, adolescents and their family choose the therapy type (low-intensity face-to-face therapy or intensive group therapy) according to their i) eligibility for the group participation, ii) will and motivation, iii) time for the scheduled group sessions (parents and adolescents) as well as the group availability. Subjects who were not eligible or did not choose the group therapy were allocated to the low-intensity face-to-face therapy arm. All patients were included in the study if they had a minimal follow-up of 5 months. Patients who had a shorter follow-up were excluded from the study.

The study was approved by the Research Ethics Committee of the HUG and adolescents and their parents provided their consents.

\section{Intensive Group Therapy}

The intensive group therapy is a family based behavioural therapy conducted by a multidisciplinary team (Contrepoids ${ }^{\circledR}$ Programme of the HUG, total $88 \mathrm{~h}$ ) was proposed to all subjects fulfilling the following criteria: i) to have at least one parent who can attend the parents' meetings, ii) to understand and speak 
Chamay-Weber et al.: Obesity Management in Adolescents: Comparison of a Low-Intensity Face-to-Face Therapy Provided by a Trained Paediatrician with an Intensive Multidisciplinary Group Therapy

French (adolescents and parents), iii) absence of psychiatric or developmental disorders limiting group participation, iv) absence of orthopaedic condition limiting the participation in physical activity.

The main goal of the intensive group therapy was to encourage lifestyle changes over a 1-year period. It consisted of psycho-educative sessions of $90 \mathrm{~min}$ with a dietician and a psychologist certified in cognitive-behavioural therapy, and sessions of $90 \mathrm{~min}$ of physical activity with a sport teacher specialised in adapted physical education. The year was divided in two parts: i) a 5-month intensive programme with one psycho-educative session per week for the adolescents and one or two sessions per month for their parents, ii) a 7-month maintenance programme with 4 sessions for the adolescents and 1 for their parents. Adolescents had a total of 18 sessions of 90 min during the year, corresponding to $27 \mathrm{~h}$ of contact with therapists, and their parents had 8 sessions in total (12 h of contact). The programme also included $90 \mathrm{~min}$ of physical activity once a week for the adolescents during the school year, except during holidays. For developmental reasons, adolescents were divided in two groups: 12-14 or 15-18 years old. A maximum of 15 families was included in each group.

The psycho-educative sessions used an integrative approach including psycho-education, stimulus control, behavioural awareness, small objectives settings and role play. The programme for parents consisted of nutrition-related topics and systemic interventions in order to facilitate family functioning [23]. In addition, each adolescent was encouraged to see the paediatrician every 3 months, during and after the end of the group therapy, for the medical monitoring.

\section{Low-Intensity Face-to-Face Therapy}

The low-intensity face-to-face therapy, which has been described elsewhere, is an out-patient consultation conducted by a paediatrician trained in obesity care [24]. Parents were encouraged to be present at all visits. Cognitive and behavioural management techniques were used to promote lifestyle changes in adolescents and their parents. The mean duration of a visit was $45 \mathrm{~min}$. Participation in structured physical activities during leisure time was strongly recommended, but not organised by the clinics. Consultations were held at an interval of 1-3 months, depending on the adolescent and family needs. There were no exclusion criteria for this type of therapy.

\section{Measures}

\section{Anthropometrics}

At each visit, body weight $(\mathrm{kg})$ and height $(\mathrm{cm})$ were measured. BMI was calculated as weight/height squared $\left(\mathrm{kg} / \mathrm{m}^{2}\right)$, and z-scores were derived using the World Health Organization references [25]. Overweight was defined as BMI z-score between 1 and 1.99 SD and obesity above 2 SD.

\section{Costs of Therapy}

Direct treatment costs were calculated on the basis of the Swiss system of procedure codes (TARMED). All costs were transformed from Swiss currency (CHF) to US Dollars (USD) using the current exchange rate (1 USD $=0.965 \mathrm{CHF}$ ). A 45-min medical consultation with a paediatrician costs USD 228.00. The Swiss health insurance companies reimburse USD 4,352.00 for the structured multidisciplinary group therapy. Noteworthy, this price has been negotiated in 2007 with the insurance companies but is underestimated [26]. In order to calculate the total direct costs of the group therapy, we cumulated the costs of the group sessions (fixed rate of USD 4,352.00) and those of the number of medical consultations before, during and after the group therapy.

\section{Statistical Analysis}

For the analysis, we selected patients who had a minimum of 5 months of follow-up, corresponding to the duration of the intensive part of the group therapy. The calculation of the sample size was based on the expected change of the BMI z-score in each group as the primary endpoint [13].

In a previous cohort study on obese children and adolescents treated with face-to-face therapy, we found a reduction of $-0.18 \pm 0.40$ of the BMI z-score [24]. Therefore, with an anticipated effect size of 0.18 , a sample size of 33 subjects in each group or sub-group could detect statistically significant differences at $\mathrm{p}<$ 
Table 1. Patients' characteristics

\begin{tabular}{lll}
\hline At inclusion & Low-intensity face-to-face therapy & Intensive group therapy \\
\hline Number, n (\%) & $159(68.2)$ & $74(31.8)$ \\
Age, years & $12.7 \pm 1.5$ & $13.9 \pm 1.7 * * *$ \\
Gender girls, n (\%) & $74(46.5)$ & $46(62.2)^{*}$ \\
BMI, kg/m ${ }^{2}$ & $28.7 \pm 4.8$ & $30.9 \pm 4.7^{* *}$ \\
BMI z-score & $2.6 \pm 0.6$ & $2.7 \pm 0.6$ \\
Number of obese subjects, n (\%) & $134(84.3)$ & $67(90.5)$ \\
\hline${ }^{*} p<0.05 ;{ }^{* *} p<0.01 ; * * * p<0.001$ between the 2 treatment arms. & \\
\hline
\end{tabular}

0.05 with a statistical power of $80 \%$. According to the number of subjects in each sub-group (the face-to-face therapy being the control group), a power calculation analysis with an alpha error of $5 \%$ showed that the statistical power to i) group allocations was $87.3 \%$, ii) the $12-14$ years age categories was $78.4 \%$; and iii) the 14-18 years age categories was $67.5 \%$. The number of subjects needed to show a difference was: i) 55 in the group and 116 in the face-to-face therapy; ii) 54 in the 12-14 years in the group and 119 in the 12-14 years face-to-face therapy; and iii) 74 in each treatment arm for the 14-18 years. Therefore, the number of subjects was enough to detect a difference between therapy allocations and age groups.

Hours of contact with a health professional were calculated as a proxy for treatment intensity. Statistical analyses were performed using the SPSS software 18.0 (Chicago, IL, USA). Data were normally distributed and presented as mean and SD. Statistical differences were analysed using independent Student's t-test, chi square test and paired t-test. Linear and standard multiple regression analyses were used to study the influence of several variables (age, gender, BMI z-score at inclusion, contact and follow-up times as well as treatment allocation) on the evolution of the BMI. Differences were considered significant if $\mathrm{p}<0.05$.

\section{Results}

Between January 2008 and June 2012, 325 adolescents aged between 11 and 18 years attended the Paediatric Obesity Clinic of the HUG. Due to a short follow-up period ( $<5$ months), 92 were excluded from this analysis (dropout rate 28.3\%). Excluded patients were comparable in age, gender and BMI-z-score at inclusion, but had $2.2 \pm 0.4 \mathrm{~h}$ of contact time and only $2.2 \pm 1.3$ consultations. Of the remaining 233 subjects, 74 met the inclusion criteria for an intensive group therapy and 159 adolescents were allocated to a face-to-face therapy.

\section{Patients' Characteristics}

A total of 120 (51\%) girls and 113 (49\%) boys were included in the study. Mean BMI and BMI z-score were $29.4 \pm 4.9 \mathrm{~kg} / \mathrm{m}^{2}$ and $2.6 \pm 0.6$, respectively. 32 subjects (14\%) were overweight and 201 (86\%) were obese. Patients' characteristics at baseline according to the treatment arm are presented in table 1 . BMI z-score at baseline was the same for the two arms.

\section{Evolution of Patients}

The group therapy was well attended by the adolescents and their family with a mean attendance rate of $84 \%$. Face-to-face and group therapies had the same mean follow-up time (table 2). Retention rate was $70.4 \%$ at 12 months, with 69/233 who stopped the follow-up at $\leq 12$ months. The retention rate was significantly better in the group therapy compared to the face-to-face therapy arm (60/74 i.e. $81.1 \%$ vs. $104 / 159$ i.e. $65.4 \%$; $p=0.015)$. Hours of contact with a therapist were 6 times higher in group than in face-to-face therapy (total before, during 
Chamay-Weber et al:: Obesity Management in Adolescents: Comparison of a Low-Intensity Face-to-Face Therapy Provided by a Trained Paediatrician with an Intensive Multidisciplinary Group Therapy

Table 2. Evolution according to age and treatment arms

\begin{tabular}{|c|c|c|c|c|c|c|}
\hline & \multicolumn{3}{|c|}{ Low-intensity face-to-face therapy } & \multicolumn{3}{|c|}{ Intensive group therapy } \\
\hline & $\begin{array}{l}12-14 \text { years } \\
(n=122)\end{array}$ & $\begin{array}{l}14-18 \text { years } \\
(n=37)\end{array}$ & $\begin{array}{l}\text { total } \\
(n=159)\end{array}$ & $\begin{array}{l}12-14 \text { years } \\
(n=42)\end{array}$ & $\begin{array}{l}14-18 \text { years } \\
(n=32)\end{array}$ & $\begin{array}{l}\text { total } \\
(\mathrm{n}=74)\end{array}$ \\
\hline Hours of contact time, $\mathrm{h}$ & $4.5 \pm 2.5$ & $4.7 \pm 2.5$ & $4.5 \pm 2.5$ & $26.0 \pm 3.9$ & $26.3 \pm 4.5$ & $26.1 \pm 4.1^{*}$ \\
\hline Follow-up time, months & $20.4 \pm 11.7^{\dagger}$ & $15.2 \pm 8.5^{\dagger}$ & $19.3 \pm 11.3$ & $20.8 \pm 9.4$ & $19.5 \pm 7.2$ & $20.2 \pm 8.5$ \\
\hline BMI z-score change & $-0.21 \pm 0.4^{¥ ¥ ¥}$ & $-0.15 \pm 0.5$ & $-0.20 \pm 0.5^{¥ ¥ ¥}$ & $-0.25 \pm 0.39^{¥ ¥ ¥}$ & $-0.23 \pm 0.67$ & $-0.24 \pm 0.5^{¥ ¥ ¥}$ \\
\hline
\end{tabular}

${ }^{*} \mathrm{p}<0.001$ between the 2 treatment arms.

${ }^{\dagger} \mathrm{p}<0.01$ between the 2 age categories in the same treatment arm.

${ }^{¥} \mathrm{p}<0.05 ;{ }^{¥} \mathrm{p}<0.01 ;{ }^{¥ ¥} \mathrm{p}<0.001$ for intra-group changes.

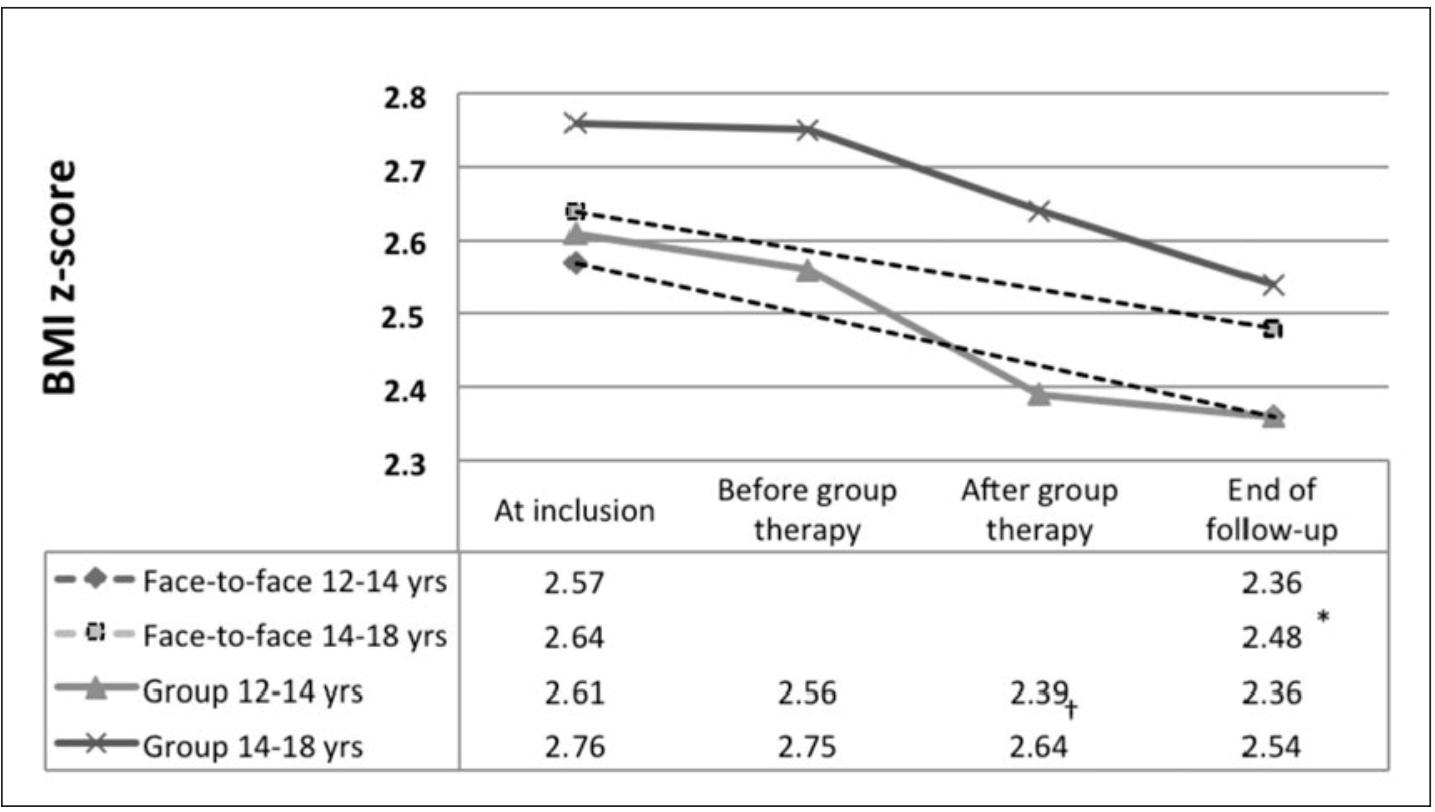

Fig. 1. BMI z-score evolution according to treatment arm and age. ${ }^{*} p<0.001$ between the inclusion and the end of follow-up. ${ }^{\dagger} \mathrm{p}<0.001$ between the beginning and the end of the group therapy.

and after the programme). BMI z-score was significantly reduced and to a similar extent in both treatment arms, even when adjusted for age and BMI ( $F=1.25 ; \mathrm{p}=0.291)$.

Changes according to the age categories (12-14 or 14-18 years old) and treatment arms are summarised in table 2. Improvements were observed for both treatments and age groups. However, changes were only significant in the younger age category in both types of therapy. In the group of 14- to 18-year-olds, BMI z-scores were also reduced but to a lesser extent. Face-to-face therapy at this age had the shorter follow-up time. Figure 1 shows the evolution of the BMI z-score according to treatment arms and age categories.

When analysed by gender, BMI z-scores were similar at baseline (boys $2.7 \pm 0.7$ vs. girls $2.5 \pm 0.6 ; p=0.072$ ). Both genders reduced significantly their BMI z-scores but globally changes were significantly greater in boys (boys $-0.30 \pm 0.57$ vs. girls $-0.13 \pm 0.34 ; p=0.008$ ), especially in the group therapy (boys $-0.49 \pm 0.7$ vs. girls $-0.09 \pm 0.3, p<0.01$ ). 
Table 3. Results of linear and multiple regression analyses for BMI z-score change

\begin{tabular}{llllll}
\hline & $\mathrm{R}^{2}$ change $\%$ & $\mathrm{~B}$ coefficient & $\mathrm{t} / \mathrm{F}$ & $95 \% \mathrm{CI}$ & $\mathrm{p}$ value \\
\hline Univariate analysis & & & & & \\
Age & -0.4 & 0.015 & 0.2 & -0.03 to 0.041 & 0.822 \\
Gender & 2.8 & -0.180 & -2.8 & -0.3 to -0.05 & $0.006^{* *}$ \\
BMI z-score at inclusion & 2.6 & -0.173 & -2.7 & -0.23 to -0.03 & $0.008^{* *}$ \\
Contact time & 0.3 & -0.087 & -1.3 & -0.01 to 0.002 & 0.184 \\
Follow-up time & 3.2 & -0.190 & -2.9 & -0.014 to -0.003 & $0.004^{* *}$ \\
Treatment allocation & -0.3 & -0.041 & -0.6 & -0.17 to 0.09 & 0.535 \\
\hline $\begin{array}{l}\text { Multivariate analysis } \\
\text { All variables }\end{array}$ & & & & & \\
Gender & 7.2 & & 7.0 & & $<0.001^{* * *}$ \\
BMI z-score at inclusion & & -0.162 & -2.5 & -0.27 to -0.04 & $0.012^{*}$ \\
Follow-up time & & -0.129 & -2.0 & -0.19 to -0.001 & $0.047^{*}$ \\
\hline
\end{tabular}

${ }^{*} \mathrm{p}<0.05,{ }^{* *} \mathrm{p}<0.01,{ }^{* * *} \mathrm{p}<0.001$.

We also categorised the evolution in 3 groups:i) improvement when BMI z-score decreased by more than 0.1 ; i) stabilisation with a BMI $z$-score change of \pm 0.1 ; and iii) poor evolution when BMI z-score increased by more than 0.1 . In our population, the majority $(52.8 \%)$ of adolescents improved their BMI z-scores (123/233 i.e., 52.8\%), 28.3\% (66/233) stabilised, and only $18.9 \%(44 / 233)$ of them show a poorer evolution. To understand which factor predicted the evolution of the BMI z-score, we performed linear and multiple regression analyses (table 3). Results of linear regression showed that gender, follow-up time as well as BMI z-score at inclusion were significantly related to changes in BMI z-score, but on little magnitude $\left(\mathrm{R}^{2}\right.$ change between 2.6 and $3.2 \%$ only). Thus, the higher the BMI z-score at inclusion and the longer the follow-up time, the greater was the BMI z-score change. It is interesting to note that age, treatment allocation or number of contact hours had no influence on BMI z-score.

The direct costs were USD 1,279.00 \pm 875.00 for face-to-face therapy and USD 6,941.00 \pm 836.00 for group therapy, the former being 5.4 times less expensive than group therapy.

\section{Discussion}

This study aimed to compare two types of interventions for obese adolescents: a lowintensity face-to-face therapy by a trained paediatrician and an intensive multidisciplinary group therapy. Results showed similar reduction in BMI z-scores in both treatment arms, suggesting that therapy by a trained paediatrician may be a good alternative to high-intensity treatment for obese adolescents. Although the reduction of BMI z-score remains modest, it was of the same magnitude, or even greater, than in previous research (BMI z-score change $-0.15)$ [27].

Among the 325 adolescents attending our obesity clinic during the study period, only 74 $(22.8 \%)$ of them were able to attend the intensive group programme. This finding highlights the difficulty to engage adolescents and their family in such a therapy. The inclusion criteria and the lack of places in group programmes were also limiting factors. Thus face-to-face therapy with a trained paediatrician was a good alternative for the patients who could not participate in an intensive group therapy. 
Interestingly, the low-intensity face-to-face therapy was as effective on BMI z-score as the intensive multidisciplinary group programme. These results contrast with most of the previousstudies reporting better outcomes for moderate to high-intensityweight management programmes $[7,9,13,28]$. To our knowledge, only few studies are supporting our findings, emphasising the importance to propose a low-intensity therapy for obesity in adolescents, especially when the load of a high-intensity therapy is not suitable for the family $[18,24]$. Furthermore, these intensive interventions are difficult to implement in primary care settings.

In this study, younger adolescents showed greater changes in BMI z-score than older ones, independently of the therapy arms. These results were not related to the BMI z-score at the time of inclusion or to the number of hours of contact. However, follow-up time was significantly longer for the 12- to 14-year-old adolescents seen in face-to-face therapy. Some other factors may explain these differences: pubertal growth spurt, changes in metabolism and nutritional needs during rapid growth and maturation, psychosocial factors, as well as parental influence at this age. The difference between age categories in the treatment arms was not significant, due to the small number of subjects.

We also observed greater BMI z-score changes among boys. Although there is still controversy in this field, three studies performed in face-to-face setting [28-30] confirm our findings, even if the difference was not significant in the study of Sabin et al. [29]. In our preceding cohort including younger children in face-to-face therapy, we did not find any association between BMI changes and gender [24]. Another study comparing face-to-face and group interventions found greater changes in girls compared to boys [31]. None of the authors addressed this issue in the discussion, probably because of the difficulty to explain this gender's difference. Greater changes in boys participating in group interventions may be due to the structured physical activities, combined with greater lean tissue mass and energy expenditure in adolescent boys. However, one study published the opposite findings in girls after a physical activity programme [32]. The authors suggested that girls may have greater benefits from the intervention being less active than boys at baseline as well as having different post-exercise eating behaviour. Further research is needed to understand genderspecific adaptations during therapy.

Societal interests request to hold down uncontrolled health care spending. Our data suggests that in adolescents a low-intensity face-to-face intervention with a trained paediatrician was far less expensive than a much more intensive programme (5.4-fold cheaper), supporting the importance of training of primary care physicians and paediatricians.

The strength of this study was the high number of adolescents who participated in the face-to-face or group therapy and the retention rate. The main limitation of this study was the prospective longitudinal design instead of a randomised controlled trial. However, due to ethical and practical reasons, it would have been difficult to impose an intensive intervention to families who could not or would not like to attend it [33]. Our study reflected real-life conditions, and not ideal circumstances of study trials with selected adolescents, which should rather be considered as strength. Another limitation was that follow-up time was not identical for all subjects, with a wide variation between them. However, the mean follow-up time was still 20 months, which is quite long considering the high dropout rate observed by most obesity centres [13].

\section{Conclusions}

This study demonstrated that a low intensity face-to-face therapy provided by a trained paediatrician was as effective as an intensive multidisciplinary group therapy to reduce the BMI z-score of adolescents with obesity. These findings highlight the need to offer diversified 
Chamay-Weber et al:: Obesity Management in Adolescents: Comparison of a Low-Intensity Face-to-Face Therapy Provided by a Trained Paediatrician with an Intensive Multidisciplinary Group Therapy

and individually adapted health care options for this population. As more than 12 million of youths are overweight in Europe and the majority of them do not have access to a specialised care centre, paediatricians and primary care physicians who received adolescents should be trained in obesity care in order to manage these patients effectively. One of the greatest challenges will be to maintain lifestyle changes in the long term, as obesity is a chronic disease that requires sustained and continuous efforts. Primary care physicians may provide this long-term follow-up, even in remote regions. More studies are however needed to assess the clinical significance and the long-term maintenance of various types of therapies on BMI in adolescents. It will also be essential to determine how to improve the knowledge and skills of paediatricians and primary care providers on obesity care at national levels.

\section{Acknowledgements}

We thank the subjects for volunteering for the study and the staff of the Paediatric Obesity Clinic of University Hospital of Geneva who supervised the group therapy and the Obesity Care Program of the Medical Direction of the University Hospital of Geneva who supported the development of the Contrepoids ${ }^{\circledR}$ group therapy.

\section{Funding Source}

This study was supported by the University Hospitals of Geneva.

\section{Disclosure Statement}

Catherine Chamay-Weber has example disclosure. The remaining authors have no financial relationships relevant to this article to disclose.

All authors have no conflicts of interest to disclose.

\section{References}

1 Lobstein T, Baur L, Uauy R: Obesity in children and young people: a crisis in public health. Obesity Rev 2004; 5(suppl 1):4-104.

2 Wang Y, Lobstein T: Worldwide trends in childhood overweight and obesity, Int J Pediatr Obes 2006;1:11-25.

-3 Must A, Strauss RS: Risks and consequences of childhood and adolescent obesity. Int J Obes Relat Metab Disord 1999;23(suppl 2):S2-11.

4 Power C, Lake JK, Cole TJ: Measurement and long-term health risks of child and adolescent fatness. Int J Obes Relat Metab Disord 1997;21:507-526.

5 Dietz WH: Periods of risk in childhood for the development of adult obesity - what do we need to learn? J Nutr 1997;127:1884S-1886S.

6 Dietz WH: Overweight in childhood and adolescence, N Engl J Med 2004;350:855-857.

-7 Hoelscher DM, Kirk S, Ritchie L, Cunningham-Sabo L: Position of the Academy of Nutrition and Dietetics: interventions for the prevention and treatment of pediatric overweight and obesity. J Acad Nutr Diet 2013;113: 1375-1394.

-8 Kirschenbaum DS, Gierut KJ: Five recent expert recommendations on the treatment of childhood and adolescent obesity: toward an emerging consensus - a stepped care approach. Child Obes 2013;9:376-385.

-9 Whitlock EP, O'Connor EA, Williams SB, Beil TL, Lutz KW: Effectiveness of weight management interventions in children: a targeted systematic review for the USPSTF. Pediatrics 2010;125:e396-418.

-10 Epstein LH, Valoski A, Koeske R, Wing RR: Family-based behavioral weight control in obese young children. J Am Diet Assoc 1986;86:481-484.

11 Epstein LH, Valoski A, Wing RR, McCurley J: Ten-year outcomes of behavioral family-based treatment for childhood obesity. Health Psychol 1994;13:373-383.

12 Nowicka P, Flodmark CE: Family therapy as a model for treating childhood obesity: useful tools for clinicians. Cl Child Psychol ad Psychiatry 2011;16:129-145. 
Chamay-Weber et al:: Obesity Management in Adolescents: Comparison of a Low-Intensity Face-to-Face Therapy Provided by a Trained Paediatrician with an Intensive Multidisciplinary Group Therapy

13 Oude Luttikhuis H1, Baur L, Jansen H, Shrewsbury VA, O'Malley C, Stolk RP, Summerbell CD: Interventions for treating obestiy in children. Cochrane Database Syst Rev 2009;1:CD001872.

14 Ho M, Garnett SP, Baur L, Burrows T, Stewart L, Neve M, Collins C: Effectiveness of lifestyle interventions in child obesity: systematic review with meta-analysis. Pediatrics 2012;130:e1647-1671.

-15 Taveras EM, Gortmaker SL, Hohman KH, Horan CM, Kleinman KP, Mitchell K, Price S, Prosser LA, Rifas-Shiman SL, Gillman MW: Randomized controlled trial to improve primary care to prevent and manage childhood obesity: the High Five for Kids study. Arch Pediatr Adolesc Med. 2011;165:714-722.

-16 Taveras EM, Hohman KH, Price SN, Rifas-Shiman SL, Mitchell K, Gortmaker SL, Gillman MW: Correlates of participation in a pediatric primary care-based obesity prevention intervention. Obesity (Silver Spring) 2011; 19:449-452.

17 McCallum Z, Wake M, Gerner B, Baur LA, Gibbons K, Gold L, Gunn J, Harris C, Naughton G, Riess C, Sanci L, Sheehan J, Ukoumunne OC, Waters E: Outcome data from the LEAP (Live, Eat and Play) trial: a randomized controlled trial of a primary care intervention for childhood overweight/mild obesity. Int J Obes (Lond) 2007; 31:630-636.

18 Saelens BE, Sallis JF, Wilfley DE, Patrick K, Cella JA, Buchta R: Behavioral weight control for overweight adolescents initiated in primary care. Obes Res 2002;10:22-32.

19 Ewing LJ, Cluss P, Goldstrohm S, Ulrich R, Colborn K, Cipriani L, Wald ER: Translating an evidence-based intervention for pediatric overweight to a primary care setting. Clinical Pediatr 2009;48:397-403.

20 Resnicow K, McMaster F, Bocian A, Harris D, Zhou Y, Snetselaar L, Schwartz R, Myers E, Gotlieb J, Foster J, Hollinger D, Smith K, Woolford S, Mueller D, Wasserman RC: Motivational interviewing and dietary counseling for obesity in primary care: an RCT. Pediatrics 2015;135:649-657.

21 Daniels SR, Hassink SG: The role of the pediatrician in primary prevention of obesity. Pediatrics 2015; 136:e275-292.

-22 Butryn ML, Wadden TA, Rukstalis MR, Bishop-Gilyard C, Xanthopoulos MS, Louden D, Berkowitz RI: Maintenance of weight loss in adolescents: current status and future directions. J Obes 2010;2010:789280.

-23 Flodmark CE, Ohlsson T, Rydén 0, Sveger T: Prevention of progression to severe obesity in a group of obese schoolchildren treated with family therapy. Pediatrics 1993;91:880-884.

24 Maggio AB, Saunders Gasser C, Gal-Duding C, Beghetti M, Martin XE, Farpour-Lambert NJ, Chamay-Weber C: BMI changes in children and adolescents attending a specialized childhood obesity center: a cohort study. BMC Pediatr 2013;13:216.

25 WHO Multicentre Growth Reference Study Group: WHO Child Growth Standards: Length/height-for-age, weight-for-age, weight-for-length, weight-for-height and body mass index-for-age: methods and development. Geneva, World Health Organization, 2006. www.who.int/childgrowth/standards/technical_report/ en/ (last accessed March 22, 2016).

26 L'Allemand D, Kirchhoff E, Bolten M, Zumbrunn A, Sempach R, Farpour-Lambert N: Evaluation du traitement des enfants et adolescents en surpoids en Suisse: analyse intermédiaire KIDSSTEP1) de la thérapie dans les programmes de groupe multiprofessionnels du 1er Mai 2012. Paediatrica 2012;23:27-30.

-27 Debar LL, Kindler L, Keefe FJ, Green CA, Smith DH, Deyo RA, Ames K, Feldstein A: A primary care-based interdisciplinary team approach to the treatment of chronic pain utilizing a pragmatic clinical trials framework. Transl Behav Med 2012;2:523-530.

28 Holm JC, Gamborg M, Bille DS, Gr Nb K HN, Ward LC, Faerk J: Chronic care treatment of obese children and adolescents. Int Journal Pediatr Obes 20116;:188-196.

29 Sabin MA, Ford A, Hunt L, Jamal R, Crowne EC, Shield JP: Which factors are associated with a successful outcome in a weight management programme for obese children? J Eval Clin Pract 2007;13:364-368.

-30 Golley RK, Magarey AM, Baur LA, Steinbeck KS, Daniels LA: Twelve-month effectiveness of a parent-led, family-focused weight-management program for prepubertal children: a randomized, controlled trial. Pediatrics 2007;119:517-525.

31 Kalavainen MP, Korppi MO, Nuutinen OM: Clinical efficacy of group-based treatment for childhood obesity compared with routinely given individual counseling. Int J Obes (Lond) 2007;31:1500-1508.

-32 Lazaar N, Aucouturier J, Ratel S, Rance M, Meyer M, Duche P: Effect of physical activity intervention on body composition in young children: influence of body mass index status and gender. Acta Paediatr 2007;96:13151320.

-33 Holm JC, Nowicka P, Farpour-Lambert NJ, O’Malley G, Hassapidou M, Weiss R, Baker JL: The ethics of childhood obesity treatment - from the Childhood Obesity Task Force (COTF) of European Association for the Study of Obesity (EASO). Obes Facts, 2014;7:274-281. 